\title{
GLOBAL EXISTENCE AND DECAY ESTIMATES FOR NONLINEAR KIRCHHOFF-TYPE EQUATION WITH BOUNDARY DISSIPATION
}

\author{
XIULI LIN AND FUSHAN LI
}

Abstract. In this paper, we consider the initial-boundary value problem for nonlinear Kirchhofftype equation

$$
u_{t t}-\varphi\left(\|\nabla u\|_{2}^{2}\right) \Delta u-a \Delta u_{t}=b|u|^{\beta-2} u,
$$

where $a, b>0$ and $\beta>2$ are constants, $\varphi$ is a $C^{1}$-function such that $\varphi(s) \geqslant \lambda_{0}>0$ for all $s \geqslant 0$. Under suitable conditions on the initial data, we show the existence and uniqueness of global solution by means of the Galerkin method and the uniform decay rate of the energy by an integral inequality.

Mathematics subject classification (2010): 35J05.

Keywords and phrases: Kirchhoff-type, initial boundary value, global existence, exponential decay.

\section{REFERENCES}

[1] M. AAssila, A. BenAiss A, Existence globale et comportement asymptotique des solutions des equations de Kirchhoff moyennement degenerees avce un terme nonlinear dissipatif, Funkcialaj Ekvacioj, 43 (2000), 309-333.

[2] M. AbDelli, A. BenAiss A, Energy decay of solutions of a degenerate Kirchhoff equation with a weak nonlinear dissipation, Nonlinear Anal., 69 (2008), 1999-2008.

[3] R. A. AdAms, Sobolev Space, New York: Academac press, 1975.

[4] F. Alabau-BoussouiRA, Decay estimates for second order evolution equations with memory, Journal of Functional Analysis, 254 (2008), 1342-1372.

[5] J.J. BAE, On uniform decay of coupled wave equation of Kirchhoff type subject to memory condition on the boundary, Nonlinear Anal., 61 (2005), 351-372.

[6] J.J. BAE, Global existence for the nonhomogeneous quasilinear wave equation with a localized weakly nonlinear dissipation in exterior domains, Acta Mathematica Scientia, 5, 29B (2009), 1203-1215.

[7] S. BERRIMI, S.A. MESSAOUDI, Existence and decay of solutions of a viscoelastic equation with a nonlinear source, Nonlinear Anal., 64 (2006), 2314-2331.

[8] M.M. Cavalcanti, A. Guesmia, General decay rates of solutions to a nonlinear wave equation with boundary conditions of memory type, Differential Integral Equations, 18 (2005), 583-600.

[9] M.M. Cavalcanti, V.N. Domingos Cavalcanti, J.S. Prates Filho, J.A. Soriano, Existence and uniform decay rates for viscoelastic problems with nonlinear boundary damping, Differential Integral Equations, 14 (2001), 85-116.

[10] M.M. Cavalcanti, V.N. Domingos Cavalcanti, P. Martinez, General decay rate estimates for viscoelastic dissipative systems, Nonlinear Anal., 68 (2008), 177-193.

[11] R.M. Christensen, Theory of viscoelasticity, Academic Press, New York, 1971.

[12] I. CHUESHOV, Long-time dynamics of Kirchhoff wave models with strong nonlinear damping, J. Differential Equations, 252 (2012), 1229-1262.

[13] L.C. Evans, Partial Differential Equations, in: Grad. Stud. Math., vol. 19, Amer. Math. Soc. Providence, RI, 1998.

[14] Q.Y. GAO, F.S. LI, Y.G. WANG, Blow-up of the solution for higher-order Kirchhoff-type equations with nonlinear dissipation, Cent. Eur. J. Math., 9, 3 (2011), 686-698.

[15] G.C. Gorain, Exponential energy decay estimates for the solutions of n-dimensional Kirchhoff type wave equation, Appl. Math. Comp., 177 (2006), 235-242. 
[16] Y.H. KAng, M.J. LeE, I.H. Jung, Stabilization of the Kirchhoff type wave equation with locally distributed damping, Appl. Math. Lett., 22 (2009), 719-722.

[17] D. KIM, Y.H. KANG, M.J. LEE, I.H. JUNG, Energy decay rate for a quasi-linear wave equation with localized strong dissipation, Computers and Mathematics with Applications, 62 (2011), 164-172.

[18] G. KIRChHOFF, Vorlesungen über Mechanik, Teubner, Leipzig, 1883.

[19] V. Komornik, Exact Controllability and Stabilization, The Multiplier Method, Res. Appl. Math., Masson/Wiley, Paris/Chichester, 1994.

[20] F.C. LI, Global existence and blow-Up of solutions for a higher-order Kirchhoff-type equation with nonlinear dissipation, Appl. Math. Lett., 17 (2004), 1409-1414.

[21] F.S. LI, Z.Q. ZHAO, Y.F. CheN, Global existence uniqueness and decay estimates for nonlinear viscoelastic wave equation with boundary dissipation, Nonlinear Analysis: Real World Applications, 12 (2011), 1759-1773.

[22] J.L. Lions, Quelques Methodes de Resolution des Problemes aux Limites Non Lineaires, Dunod, Paris, 1969.

[23] A.T. Louredo, M. Milla Miranda, Local solutions for a coupled system of Kirchhoff-type, Nonlinear Anal., 74 (2011), 7094-7110.

[24] L. LU, S. LI, S. ChAI, On a viscoelastic equation with nonlinear boundary damping and source terms: Global existence and decay of solution, Nonlinear Analysis: Real World Applications, 12 (2011), 295-303.

[25] S.A. Mess AOUdi, B.S. Houari, A blow-up result for a higher-order nonlinear Kirchhoff-type hyperbolic equation, Appl. Math. Lett., 20 (2007), 866-871.

[26] M. NAKAO, An attractor for a nonlinear dissipative wave equation of Kirchhoff type, J. Math. Anal. Appl., 353 (2009), 652-659.

[27] K. Nishinara, Y. YAmada, On global solutions of some degenerate quasilinear hyperbolic equations with dissipative terms, Funkcialaj Ekvacioj, 33 (1990), 151-159.

[28] K. ONO, Global existence, decay and blowup of solutions for some mildly degenerate nonlinear Kirchhoff strings, Journal of Differential Equations, 137 (1997), 273-301.

[29] K. ONO, On decay properties of solutions for degenerate strongly damped wave equations of Kirchhoff type, J. Math. Anal. Appl., 381 (2011), 229-239.

[30] K. ONO, K. NishinARA, On a nonlinear degenerate integro-differential equation of hyperbolic type with a strong dissipation, Advances in Mathematics Seciencesn and Applications, 5 (1995), 457-476.

[31] J. Simon, Compact sets in the space $L^{p}(0, T ; B)$, Ann. Mat. Pura. Appl, (IV) CXLVI (1986), 65-96.

[32] T. TANIGUCHI, Existence and asymptotic behaviour of solutions to weakly damped wave equations of Kirchhoff type with nonlinear damping and source terms, J. Math. Anal. Appl., 361 (2010), 566-578.

[33] Y.J. YE, On the exponential decay of solutions for some Kirchhoff-type modelling equations with strong dissipation, Applied Mathematics, 1 (2010), 529-533.

[34] E. Vitillaro, Global existence for the wave equation with nonlinear boundary damping and source terms, J.Differential Equations, 186 (2002), 259-298.

[35] S.T. WU, Asymptotic behavior of solutions for nonlinear wave equations of Kirchhoff type with a positivenegative damping, Appl. Math. Lett., 25 (2012), 1082-1086.

[36] S.T. WU, Exponential energy decay of solutions for an integro-differential equation with strong damping, J. Math. Anal. Appl., 364 (2010), 609-617.

[37] S.T. Wu, On decay and blow-up of solutions for a system of nonlinear wave equations, J. Math. Anal. Appl., 394 (2012), 360-377.

[38] S.T.WU, L.Y. TSAI, Blow-up of solutions for some non-linear wave equations of Kirchhoff type with some dissipation, Nonlinear Anal., 65 (2006), 243-264.

[39] Z.J. YANG, Y.Q. WANG, Global attractor for the Kirchhoff type equation with a strong dissipation, J. Differential Equations, 249 (2010), 3258-3278.

[40] Z.J. YANG, X. LI, Finite-dimensional attractors for the Kirchhoff equation with a strong dissipation, J. Math. Anal. Appl., 375 (2011), 579-593. 